Ophthalmologe 2022 · 119:221-222

https://doi.org/10.1007/s00347-021-01530-1 Angenommen: 20. Oktober 2021

(c) The Author(s), under exclusive licence to Springer Medizin Verlag $\mathrm{GmbH}$, ein Teil von Springer Nature 2021

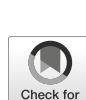

\title{
UV-Schutz am Auge - ein häufig vernachlässigtes Thema
}

\author{
Vinodh Kakkassery ${ }^{1}$. Ludwig M. Heindl ${ }^{2,3}$ \\ ${ }^{1}$ Klinik für Augenheilkunde, Universitätsklinikum Schleswig-Holstein, Campus Lübeck Universität zu \\ Lübeck, Lübeck, Deutschland \\ ${ }^{2}$ Zentrum für Augenheilkunde, Medizinische Fakultät und Universitätsklinikum Köln, Universität zu Köln, \\ Köln, Deutschland \\ ${ }^{3}$ Center for Integrated Oncology (CIO) Aachen-Bonn-Köln-Düsseldorf, Köln, Deutschland
}

Neben der Versorgung von pathologischen Prozessen ist die Prävention von Erkrankungen am Auge ureigenste Aufgabe der Gemeinschaft von Augenärzt:innen. Gerade die Fachgesellschaften mit ihren Fachgremien sind geeignet, evidenzbasiertes Wissen zu identifizieren, Empfehlungen für die Patient:innen, aber auch für die Behandler:innen zu formulieren und den weiteren Bedarf an neuer Forschung zu identifizieren. In diesem Zusammenhang ist der interdisziplinäre Austausch mit anderen medizinischen Disziplinen, aber auch mit weiteren wissenschaftlichen Kreisen und mit Patientenvertreter:innen unerlässlich.

In diesem Kontext werden die potenziellen gesundheitlichen UV-Strahlenschäden von dem fächerübergreifenden UVSchutz-Bündnis, das vom Bundesamt für Strahlenschutz koordiniert wird, analysiert und bewertet. Die aktuelle Situation zeigt, dass wissenschaftlich erwiesenermaßen UV-Strahlung auch am Auge zur Induktion von benignen und malignen Erkrankungen führen kann. Allerdings hat dieses Wissen bisher nur teilweise die Bevölkerung erreicht, sodass der notwendige eigene UV-Schutz noch keine Selbstverständlichkeit ist.

\section{I) UV-Strahlung kann am Auge zur Induktion von benignen und malignen Erkrankungen führen}

Die Deutsche Ophthalmologische Gesellschaft (DOG) sowie seit Neuestem der Be- rufsverband der Augenärzte (BVA) sind aktive Mitglieder des UV-Schutz-Bundes. Die Autoren Vinodh Kakkassery und Ludwig M. Heindl dürfen diese beiden Fachgesellschaften im UV-Schutz-Bündnis vertreten. Aktuell möchten wir mit diesem Leitthema die wichtige Thematik gesteigert in den Fokus der Augenärzt:innen bringen, um weiter zu sensibilisieren. Dr. Baldermann hat in diesem Zusammenhang einen mit allen Mitglieder:innen des UV-Schutz-Bündnisses abgestimmten Beitrag verfasst, der das Gremium vorstellt und die Ziele sowie Weiteres erläutert [1]. Die Arbeitsgruppe um Prof. Dr. Schuster hat den aktuellen Wissensstand bezüglich der UV-Strahlenschäden im vorderen Augenabschnitt zusammengefasst [2]. Die Arbeitsgruppe um PDDr. Thomas Ach hat im Weiteren einen Beitrag zum Wissen um die UV-Strahlenschäden des hinteren Augenabschnitts erstellt [3]. Abgerundet wird das Leitthema durch eine schematische Darstellung des UV-Schutz-Bündnisses im Zeitstrahl [4].

Der folgerichtige nächste Schritt ist dann die Sensibilisierung der Bevölkerung bezüglich der UV-Strahlenschäden sowie des sinnvollen UV-Schutzes eines/ $r$ jeden Einzelnen. Im Weiteren ist es natürlich auch das Ziel, mit weiteren Forschungsprojekten die potenziellen Risiken von UV-Strahlen auch am Auge weiter zu erfassen und daraus weitere Handlungsempfehlungen abzuleiten.

Es ist und bleibt eine Frage der Zeit, bis Sie als Augenarzt/ärztin vermehrt von Ihren Patient:innen nach dem richtigen 


\section{Zur Einführung zum Thema}

UV-Schutz gefragt werden. Wir hoffen, mit diesen Beiträgen und der zukünftigen Arbeit in den Fachgesellschaften hier ein wenig Hilfestellung leisten zu können.

Mit kollegialen Grüßen aus Lübeck und Köln

Ihr Vinodh Kakkassery und Ludwig M. Heindl

\section{Korrespondenzadresse}

PD. Dr. med. Vinodh Kakkassery

Klinik für Augenheilkunde, Universitätsklinikum Schleswig-Holstein, Campus Lübeck Universität zu Lübeck

Ratzeburger Allee 160, 23538 Lübeck, Deutschland

vinodh.kakkassery@gmail.com

Interessenkonflikt. V. Kakkassery und L.M. Heindl geben an, dass kein Interessenkonflikt besteht.

\section{Literatur}

1. Baldermann C, UV-Schutz-Bündnis (2021) Das UVSchutz-Bündnis in Deutschland - Zweck und Ziele Ophthalmologe, in press

2. Hampel U, Elflein HM, Kakkassery V, HeindI LM Schuster AK (2021) UV-Strahlenexpositionsbedingte Veränderungen am vorderen Augenabschnitt. Ophthalmologe, in press

3. Saßmannshausen M, Ach T (2021) Einfluss von ultravioletter Strahlung auf die Netzhaut. Ophthalmologe. https://doi.org/10.1007/s00347021-01506-1

4. Baldermann C, UV-Schutz-Bündnis (2021) Das UVSchutz-Bündnis in Deutschland - Zeitstrahl der inhaltlichen Entwicklung. Ophthalmologe, in press

\section{UV-Schäden am Auge}

Eine Übersicht

Urheber @Vinodh Kakkassery (Lübeck)

Herstellung Harald Stephan (Herne) 